\title{
Urinary System Findings Link Identifier
}

National Cancer Institute

\section{Source}

National Cancer Institute. Urinary System Findings Link Identifier. NCI Thesaurus. Code C162026.

A sequence of characters used as a linkage between related urinary system findings identifier records. 